\title{
Effects of temporal and spatial separation on velocity and strength of illusory line motion
}

\author{
Timothy L. Hubbard • Susan E. Ruppel
}

Published online: 13 January 2011

(C) Psychonomic Society, Inc. 2011

\begin{abstract}
The effects of line length and of spatial or temporal distance on illusory line motion (i.e., on the perception that a stationary line unfolds or expands away from a previously presented stationary cue) were examined in five experiments. Ratings of relative velocity decreased with increases in stimulus onset asynchrony between appearance of the cue and appearance of the line (from 50 to $450 \mathrm{~ms}$ ), whereas the extremity of ratings of direction (i.e., strength of the ratings of illusory line motion) increased with increases in stimulus onset asynchrony (from 50 to either 250 or $450 \mathrm{~ms}$ ). Ratings of relative velocity increased with increases in line length, whereas ratings of direction were not influenced by increases in line length. Ratings of relative velocity and direction were not influenced by increases in the distance of the near or the far end of the line from the cue. Implications of these data for attentional theories and apparent-motion theories of illusory line motion are discussed.
\end{abstract}

Keywords Illusory line motion · Attention · Apparent motion $\cdot$ Perception of velocity $\cdot$ Dynamics

In illusory line motion (ILM; also known as the line motion effect, line motion illusion, shooting line illusion, and motion induction), a cue appears, and shortly thereafter, a

T. L. Hubbard $(\bowtie)$

Department of Psychology, Texas Christian University,

TCU Box 298920, Fort Worth, TX 76129, USA

e-mail: timothyleehubbard@gmail.com

S. E. Ruppel

Department of Psychology, University of South Carolina Upstate, Spartanburg, SC 29303, USA stationary line appears (e.g., Downing \& Treisman, 1997; Fuller \& Carrasco, 2009; Hikosaka, Miyauchi, \& Shimojo, 1993a). The entirety of the line is presented simultaneously, but observers perceive the line to be presented sequentially, such that the line appears to "unfold" or "be drawn" from the near end of the line (i.e., the end closest to the cue) to the far end (i.e., the end most distant from the cue). There is no actual or implied motion in the display, but observers perceive the far end of the line as moving away from the cue as that line is perceived to extend or expand away from the cue. Surprisingly, accounts of ILM typically do not explicitly address the perceived velocity of ILM, although some studies have used matching or cancellation of the perceived velocity of ILM as an investigative tool (e.g., Fuller \& Carrasco, 2009, determined the point of subjective equality between a line exhibiting ILM and a line sequentially drawn; von Grünau, Racette, \& Kwas, 1996, used differences in luminance to cancel the perception of motion in an ILM stimulus). The present study examined how the perceived velocity of ILM and the perceived direction and strength of ILM were influenced by differences in the temporal separation and spatial separation of the cue and line.

The most common account of ILM involves differences in the initial strength of attention at different points along the line (e.g., Bavelier, Schneider, \& Monacelli, 2002; Hikosaka et al., 1993a; Shimojo, Hikosaka, \& Miyauchi, 1999). According to this view, attending to the cue produces an attentional gradient such that portions of the line closer to the cue receive a greater allocation of attention, and so are processed more quickly than are portions of the line more distant from the cue. As a result, portions of the line closer to the cue enter into perceptual awareness more rapidly than do portions more distant from the cue (i.e., prior entry; Spence \& Parise, 2010), so that the 
line is perceived to unfold or extend from the initially perceived location closest to the cue toward the subsequently perceived location most distant from the cue. The cue does not have to be in the same modality as the visual line (Shimojo, Miyauchi, \& Hikosaka, 1997). Both exogenous attention and endogenous attention have been suggested to contribute to ILM (e.g., von Grünau, Dubé, \& Kwas, 1996; Schmidt, 2000), but at least one report has suggested that endogenous attention does not contribute to ILM (Christie \& Klein, 2005). If the line is more distant from the cue or if the line is longer, the gradient will be stronger (i.e., differences between the cue and the far end of the line will be larger), which would predict a greater strength of ILM (but see Scharlau \& Horstmann, 2006) and a faster perceived velocity of ILM.

A second notion involving attention and ILM is that additional attention is automatically reallocated (shifted) to the far end of the line following ILM. Hamm and Klein (2002) found that detection of a change in the shape of a target (e.g., from a square to an elongated rectangle) was facilitated if the target was at the far end of a line that had just exhibited ILM rather than at the far end of a line that had not just exhibited ILM. Interestingly, facilitation of a target at the far end of a line that had just exhibited ILM did not differ from facilitation of a target at the near (cued) end of a similar line, and this suggested that attention was not reallocated from the near to the far end of the line. Hamm and Klein suggested that their results were consistent with a zoom lens model of attention (see, e.g., Eriksen, Pan, \& Botella, 1993), in which ILM stretches the attentional "zoom lens" to encompass the moving line without disengaging from the initially cued location. This suggests a dynamic expansion of attention after ILM, whereas an attentional-gradient account posits that a relatively static distribution of attention gives rise to ILM. If ILM attracts attentional resources to the far end of the expanding line, the rate of stretching of the zoom lens of attention might mirror the perceived velocity of expansion of the line. Given that judgments of a target at the far end of the line were facilitated as much as judgments of a target at the near (cued) end, no differences in strength of ILM as a function of line length would be predicted.

Another account of ILM involves apparent motion (e.g., Downing \& Treisman, 1997; Kawahara, Yokosawa, Nishida, \& Sato, 1996). According to this view, impletion processes involved in apparent motion bind successive presentations of the cue and the line into a representation of a single object. If participants perceive apparent motion between the cue and the line, it could be predicted that extending the spatial distance between the cue and the line or decreasing the temporal distance (i.e., decreasing the latency) between the appearance of the cue and the appearance of the line might influence the perceived velocity of ILM (if apparent motion still occurred). However, Eagleman and Sejnowski (2003) spatially separated the cue and the line in an ILM display by as much as $6^{\circ}$ of arc (well beyond the distance at which translational apparent motion could occur) and still obtained ILM; based on this result, they suggested that ILM does not result from apparent motion. Although most discussions of apparent motion and ILM involve translational apparent motion, it is possible that ILM might instead involve transformational apparent motion. In transformational apparent motion, participants are successively shown different shapes, and they perceive a smooth transformation between those shapes (e.g., Tse \& Logothetis, 2002). Discussions of transformational apparent motion have not addressed ILM, but it is possible that ILM might result from the cue being perceived to transform into a line rather than from the expansion of a line distinct from the visual cue.

Several researchers have addressed the strength of ILM (e.g., Christie \& Klein, 2005; Faubert \& von Grünau, 1995; von Grünau \& Faubert, 1994; Schmidt, 2000); in these investigations, strength of ILM appeared to reflect the vividness of a sense of motion of the line, and the relationship between perceived strength and perceived velocity of ILM was not explicitly considered. Furthermore, accounts of ILM involving an attentional gradient, reallocation of attention, or apparent motion do not make consistent predictions regarding (1) how the velocity of ILM is influenced by the distance of the line from the cue or (2) the relationship between the perceived velocity of ILM and the perceived strength of the motion in ILM. Although it might initially seem that a stronger sense of motion in ILM should result from a faster velocity, some researchers have suggested that a stronger sense of motion in ILM should result from a slower perceived velocity (e.g., Christie \& Klein, 2005, suggested that a stronger motion experience results from a slower drawing speed). Also, factors not related to velocity per se (e.g., luminance and color contrast; von Grünau \& Faubert, 1994) can influence the strength of motion perception in ILM. Even so, given that ILM involves a change in the perceived location of the (far end of the) line over time, the perceived velocity of that change is a potentially important element of ILM and might be related either to the strength (or other characteristics) of ILM or to other aspects of the cue and line in important ways.

The experiments reported here collected ratings of the relative perceived velocity of expansion of the line in ILM and ratings of the perceived direction (and relative strength) of expansion of the line. Rather than using a dichotomous measure for ratings of direction (as had often been used in previous studies), a multivalued scale was used, and more 
extreme values (i.e., those closer to the endpoints) on this scale would reflect stronger ILM (cf. Christie \& Klein, 2005). Examination of ratings of perceived velocity and perceived direction singly and in combination should help reveal characteristics of the perceived velocity and perceived strength of ILM, as well as the relationship between the perceived velocity and perceived strength. Experiment 1 varied the stimulus onset asynchrony (SOA) between when the cue appeared and when the line appeared and kept the length of the line and the distance of the line from the cue constant. Experiment 2 varied length of the line and kept distance from the cue of the near end of the line (i.e., the end closest to the cue) and SOA constant. Experiment 3 varied distance from the cue of the near end of the line and kept length of the line and SOA constant. Experiment 4 varied length of the line and distance of the near end of the line from the cue and kept distance from the cue of the far end of the line (i.e., the end most distant from the cue) and SOA constant. Experiment 5 varied length of the line and the distances from the cue of the near and the far end of the line and kept SOA constant.

\section{Experiment 1}

Experiment 1 presented an ILM display similar to that in Downing and Treisman (1997); a schematic of this display is shown in Fig. 1. First, a fixation point appeared horizontally centered in the bottom half of the display. A cue then appeared in the upper left or upper right, and after a brief delay, a horizontal line appeared to the right or left of the cue, respectively, and vertically aligned with the cue. After a brief delay, the display cleared, and a scale for rating the perceived velocity of (illusory) motion or the perceived direction and strength of (illusory) motion appeared. The results of previous studies suggested that ILM is stronger when the cue precedes the line by 100 300 ms (e.g., Bavelier et al., 2002; Hikosaka et al., 1993a), so if the perceived velocity of ILM reflects the strength of the perceived expansion of ILM, it could be predicted that ratings of perceived velocity should be higher with an SOA of $100-300 \mathrm{~ms}$ than with a smaller or larger SOA. Alternatively, if the perceived velocity of ILM is separate and distinct from the strength of ILM, it is not clear whether ratings of perceived velocity would necessarily parallel ratings of the strength of ILM. Accordingly, the SOA between appearance of the cue and appearance of the line in Experiment 1 was 50,250 , or $450 \mathrm{~ms}$. In one block of trials, participants rated perceived relative velocity of the expansion of the line, and in another block of trials, they rated perceived direction and relative strength of the expansion of the line.

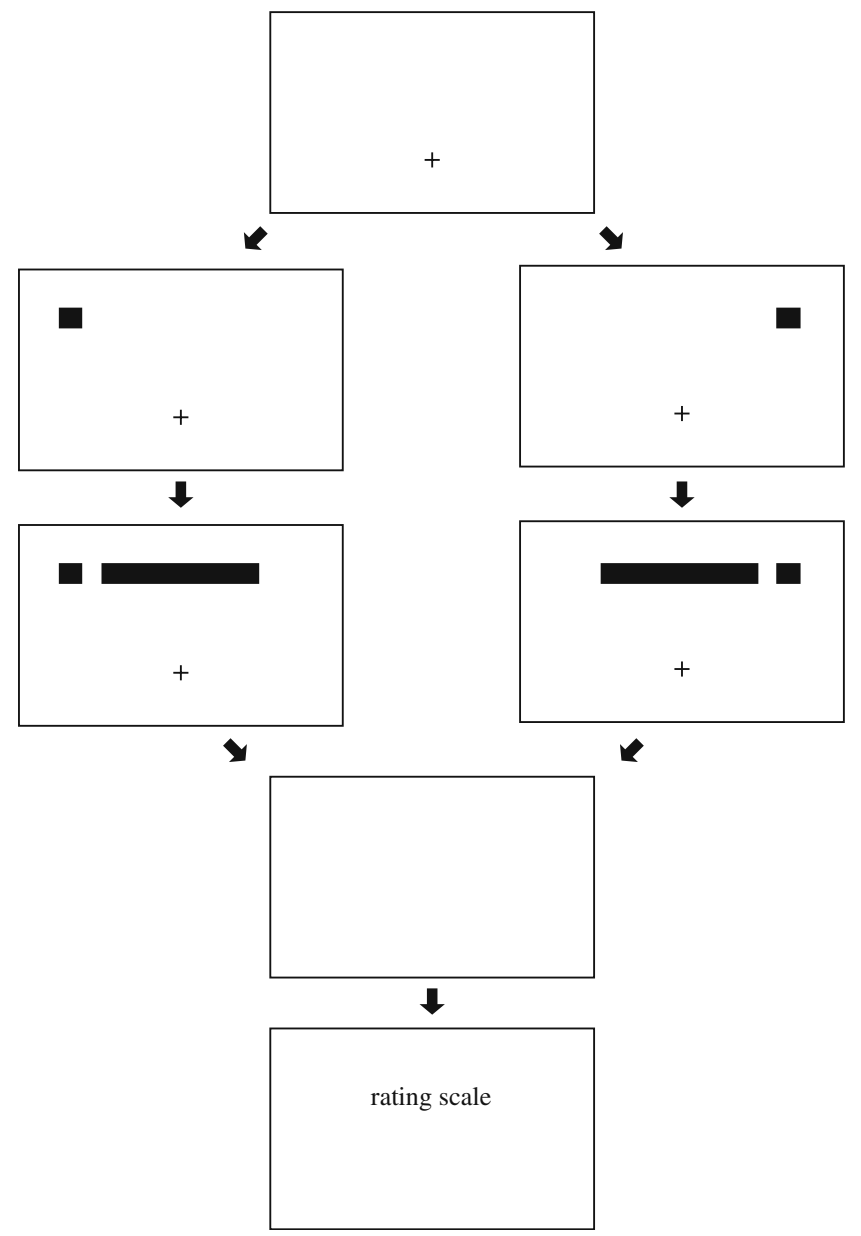

Fig. 1 The structure of a trial in Experiment 1. A fixation point is presented (first/top row), then a cue appears on either the left or right side (second row). A line is then presented (third row). The cue and line vanish (fourth row), and a rating scale appears on the display; the observers rate either velocity or direction (fifth/bottom row)

\section{Method}

Participants The participants were 20 undergraduates from the University of South Carolina, Upstate, who received partial course credit and were naïve to the hypotheses.

Apparatus The stimuli were displayed upon and the data were collected with a Gateway desktop computer equipped with a 15 -in. color monitor with a refresh rate of $60 \mathrm{~Hz}$ and a resolution of $1,024 \times 768$ pixels. Participants' head and eye movements were not constrained, and the average viewing distance was approximately $60 \mathrm{~cm}$.

Stimuli The cue was a black square 20 pixels in width and height (approximately $0.83^{\circ}$ ), the line was a black rectangle 196 pixels in width and 20 pixels in height (approximately $8.13 \times 0.83^{\circ}$ ), and the cue and line were presented on a white background; the luminance of the cue and of the line was $1.9 \mathrm{~cd} / \mathrm{m}^{2}$, and the luminance of the background was 
$103.0 \mathrm{~cd} / \mathrm{m}^{2}$. The cue and the line were vertically aligned and located slightly above the vertical midpoint of the display. There was a separation of 20 pixels $\left(0.83^{\circ}\right)$ of empty space between the closest vertical edges of the cue and of the line. The SOA between appearance of the cue and appearance of the line was 50,250 , or $450 \mathrm{~ms}$. The fixation point was a plus shape, and each of its four arms was 10 pixels in length (i.e., the plus shape was 20 pixels in width [the same width as the cue] and 20 pixels in height [the same height as the cue and the line]). The fixation point was at the horizontal center of the display and was approximately one-third of the vertical distance from the bottom to the top of the display. Ratings of perceived velocity were made using a 1-7 scale (in which 1 was very slow and 7 was very fast), and ratings of perceived direction (and strength) were made using a 1-7 scale (in which 1 was clearly expanded from the left, 4 was appeared all at once, and 7 was clearly expanded from the right). ${ }^{1}$ In one block of trials, participants rated perceived relative velocity of (illusory) motion, and in another block, they rated perceived direction (and relative strength) of (illusory) motion. In each block, each participant received 42 trials (2 [cue: left, right] $\times 3$ [SOA: 50, 250, $450 \mathrm{~ms}] \times 7$ [replications]) in a different random order.

Procedure The rating task was blocked, with ratings of perceived velocity collected in one block and ratings of perceived direction (and strength) collected in another block. The order of the blocks was counterbalanced across participants. Before beginning each block, participants were given a practice session consisting of 10 practice trials randomly drawn from the experimental trials for that block. When participants were ready for a trial to begin, they pressed a designated key. The cue immediately appeared, and the line appeared 50, 250, or $450 \mathrm{~ms}$ later. The cue and line were visible for an additional $250 \mathrm{~ms}$, and then they simultaneously vanished. After a $250-\mathrm{ms}$ pause, a rating scale appeared centered in the display and remained visible until the participant responded. In the velocity block, only the velocity rating scale appeared; in the direction block, only the direction rating scale appeared. In both blocks, ratings were entered by pressing the appropriate keys on a numeric keypad. After the participant entered a rating,

\footnotetext{
${ }^{1}$ It might initially appear that the velocity scale and the direction scale are not equivalent, as the "zero" or "null" point is at the end of the velocity scale and the midpoint of the direction scale. However, each scale allows the participants to choose which of seven options best categorizes their perception of the stimulus (and seven response categories is usually considered an optimal number; e.g., Hofmans, Theuns, \& Mairesse, 2007; Weijters, Cabooter, \& Schillewaert, 2010). Furthermore, the format of the rating scales allows us to combine a rating scale for direction and strength modeled after that of Christie and Klein (2005) with a measure of perceived relative velocity that contains the same number of categories.
}

the display cleared and a prompt to begin the next trial appeared.

\section{Results}

Ratings of perceived velocity are shown in the top panel of Fig. 2, and ratings of perceived direction are shown in the bottom panel of the figure. Preliminary analyses revealed that block order did not influence the ratings, so subsequent analyses were collapsed across block orders.

Velocity Ratings of perceived velocity were analyzed in a 2 (cue) $\times 3(\mathrm{SOA})$ repeated measures ANOVA. Neither cue, $F(1,19)=0.11, M S E=0.21, p>.74$, nor Cue $\times$ SOA, $F(2,38)=1.72, M S E=0.13, p>.19$, was significant. SOA was highly significant, $F(2,38)=73.09, M S E=1.72, p<$ .0001 , and a least-squares comparison revealed that all pairwise comparisons between the 50-ms $(M=6.58)$, 250-ms $(M=4.51)$, and 450-ms $(M=3.05)$ SOAs were significant.

Direction Ratings of perceived direction were analyzed in a 2 (cue) $\times 3(\mathrm{SOA})$ repeated measures ANOVA. Cue, $F(1,19)=193.66, M S E=2.76, p<.0001$, and Cue $\mathrm{x}$ SOA, $F(2,38)=18.80, M S E=0.63, p<.0001$, were significant. When cues were on the left, lines were more
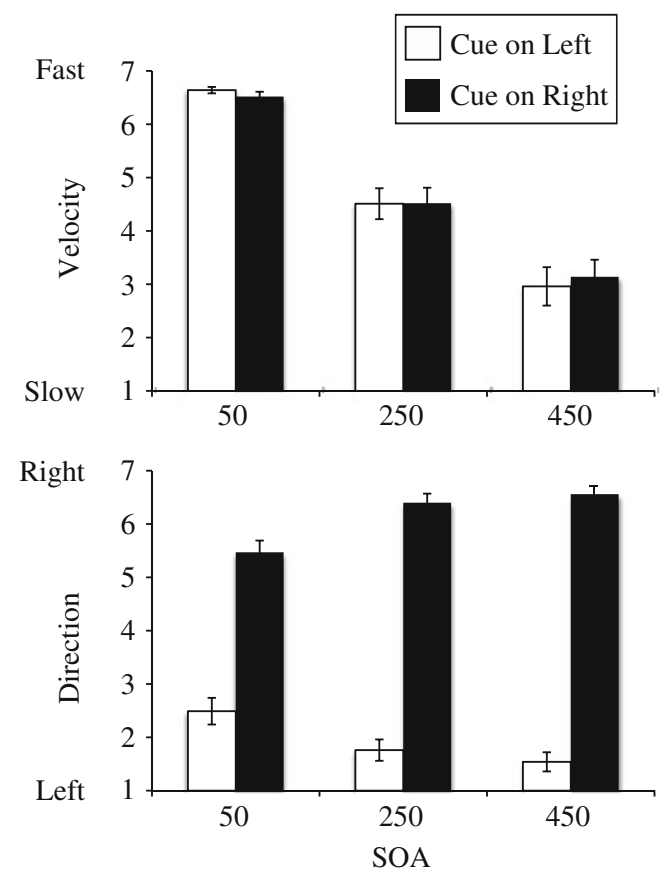

Fig. 2 The ratings of velocity and direction as a function of SOA in Experiment 1. Data for velocity ratings are shown in the top panel, and data for direction ratings are shown in the bottom panel. Data when the cue is on the left or right are indicated by open and filled columns, respectively. Error bars reflect standard errors of the means 
likely to be rated as expanding from the left $(M=1.93)$, and when cues were on the right, lines were more likely to be rated as expanding from the right $(M=6.14)$. To explore the Cue $\mathrm{x}$ SOA interaction further, a difference score (mean rating for right cue minus mean rating for left cue) was calculated for each SOA for each participant, and these difference scores were analyzed in a one-way repeated measures ANOVA. Difference score was significant, $F(2,38)=18.80, M S E=1.25, p<.0001$, and leastsquares comparison revealed that the $50 \mathrm{~ms}$ SOA $(M=$ 2.98) resulted in smaller difference scores (i.e., less extreme ratings) than did the 250 -ms $(M=4.64)$ or the 450 -ms $(M=5.02)$ SOA, and difference scores for the 250- and 450-ms SOAs did not significantly differ. As shown in the bottom panel of Fig. 2, the difference in ratings of direction for cues on the left and for cues on the right was less when the SOA was $50 \mathrm{~ms}$ than when the SOA was 250 or $450 \mathrm{~ms}$ (i.e., ratings of direction were more extreme when SOA was 250 or $450 \mathrm{~ms}$ ). SOA was not significant, $F(2,38)=0.65, M S E=0.17, p>.52$.

Ratings of perceived direction for each condition were tested against a null rating of 4 (i.e., against a rating of appeared all at once, Bonferroni correction of $.05 / 6=$ .0083). When cues were on the left for SOAs of $50 \mathrm{~ms}(M=$ 2.49), $t(19)=-6.10, p<.0001,250 \mathrm{~ms}(M=1.76), t(19)=$ $-11.38, p<.0001$, and $450 \mathrm{~ms}(M=1.54), t(19)=-13.45$, $p<.0001$, ratings were significantly less than 4 , indicating that lines were perceived as expanding from the left. When cues were on the right for SOAs of $50 \mathrm{~ms}(M=5.47), t(19)=$ $6.60, p<.0001,250 \mathrm{~ms}(M=6.40), t(19)=14.14, p<.0001$, and $450 \mathrm{~ms}(M=6.56), t(19)=17.33, p<.0001$, ratings were significantly greater than 4 , indicating that lines were perceived as expanding from the right.

\section{Discussion}

Ratings of the perceived velocity of ILM decreased with increases in the SOA between when the cue and the line appeared. Distance between the cue and the line was constant across different SOAs, so the decrease in ratings of perceived velocity with increases in SOA cannot be due to differences in distance. One speculative possibility is that the cue is the source of ILM (e.g., perhaps as a form of polarized gamma motion; cf. Kanizsa, 1951) and that the length of time required to move from the cue to the line (i.e., the SOA) influences the subsequent perceived velocity of ILM (e.g., for a given distance, a shorter SOA would require a faster velocity, so the perceived velocity of expansion of the line following a shorter SOA would be faster). With a constant SOA, such a notion predicts that lines with a near edge farther from the cue should exhibit a faster perceived velocity of ILM than would lines with a near edge closer to the cue, and this notion is examined further in Experiments 3, 4, and 5. However, it is not clear how such an account is consistent with the occurrence of ILM when the cue and the line are spatially overlapping (e.g., as in Hikosaka et al., 1993a). The decrease in ratings of perceived velocity with increases in SOA in Experiment 1 suggests that with a sufficiently long SOA, perceived velocity would decrease to zero, and this is consistent with previous claims suggesting that the strength of ILM decreases with even longer SOAs (e.g., Bavelier et al., 2002).

An increase in the perceived strength of ILM would be reflected by a lower rating of perceived direction when the cue was on the left and a higher rating of perceived direction when the cue was on the right; therefore, any effect of SOA on the intensity of ILM would be revealed in a Cue $\mathrm{x}$ SOA interaction in ratings of direction rather than by a cue main effect. Ratings of perceived direction did exhibit a significant Cue x SOA interaction, such that ratings of perceived direction were more extreme with an SOA of 250 or $450 \mathrm{~ms}$ than with an SOA of $50 \mathrm{~ms}$. This pattern is consistent with Christie and Klein's (2005) notion that a slower drawing speed (i.e., a slower perceived velocity of expansion) is linked with a stronger sense of motion, as the Cue x SOA interaction in ratings of perceived direction revealed a stronger ILM (i.e., more extreme ratings) with an SOA of 250 or $450 \mathrm{~ms}$ than with an SOA of $50 \mathrm{~ms}$. This pattern is also consistent with previous findings that the perceived strength of ILM increases with increases in cue lead time (for SOAs under half a second), and this gives greater confidence that ILM similar to that found in previous reports was experienced by participants in Experiment 1. Finally, the significant Cue $\mathrm{x}$ SOA interaction in ratings of perceived direction and strength, as well as the significant effect of SOA on ratings of perceived velocity, underscore that ILM is a dynamic process that unfolds across time as well as across space.

\section{Experiment 2}

With a constant perceived velocity of expansion, a long line would require more time to unfold or extend than would a short line, and given that Experiment 1 found that the perceived velocity of ILM decreases with increases in the time between the appearance of the cue and appearance of the line, it could be predicted that a long line would exhibit a slower overall average perceived velocity than would a short line (as averaging over a larger total decrease in velocity would result in a smaller average overall velocity). Alternatively, if ILM is of a limited duration regardless of the perceived velocity of illusory motion, it could be predicted that the perceived velocity of ILM would be faster when the line is longer (i.e., within a given unit of 
time, a long line would have to unfold more rapidly to reach its full extent than would a short line). Given that a longer line would have to exhibit a faster perceived velocity to extend across a longer distance, it could be predicted that a long line would exhibit a faster overall average perceived velocity than would a short line. Accordingly, in Experiment 2 the length of the line was varied. It is not clear whether line length would have any effect on ratings of the direction (and strength) of perceived expansion, although Christie and Klein's (2005) notion that a slower drawing speed is linked with a stronger sense of motion predicts more extreme ratings of perceived direction for a given line length that is perceived to expand at a slower velocity.

Method

Participants The participants were 19 undergraduates from the same participant pool used in Experiment 1, and none had participated in Experiment 1.

Apparatus The apparatus was the same as in Experiment 1.

Stimuli The cue and line stimuli were the same as in Experiment 1, with the following exceptions: On all trials, the SOA was $250 \mathrm{~ms}$. The length of the line could be short (96 pixels, $3.98^{\circ}$ ), medium (146 pixels, $6.06^{\circ}$ ), or long (196 pixels, $8.13^{\circ}$; same as in Experiment 1). The distance of the near end of the line from the cue was the same in all trials (20 pixels, $0.83^{\circ}$; same as in Experiment 1 ), but the length of the line and the distance of the far end of the line from the cue varied across trials (see Fig. 3). Rating scales were the same as in Experiment 1. In each block, each participant received 42 trials (2 [cue: left, right] $\times 3$ [length: short, medium, long] $\times 7$ [replications] in a different random order.

Procedure The procedure was the same as in Experiment 1, with the following exception: On each trial, the SOA between the cue and the line was always $250 \mathrm{~ms}$.

\section{Results}

Ratings of perceived velocity are shown in the top panel of Fig. 4, and ratings of perceived direction are shown in the bottom panel of the figure. Preliminary analyses revealed that block order did not influence ratings, so subsequent analyses were collapsed across block orders.

Velocity Ratings of perceived velocity were analyzed in a 2 (cue) $\times 3$ (length) repeated measures ANOVA. Neither cue, $F(1,18)=0.11, M S E=0.10, p>.51$, nor Cue $\times$ Length, $F(2,36)=0.90, M S E=0.14, p>.91$, was

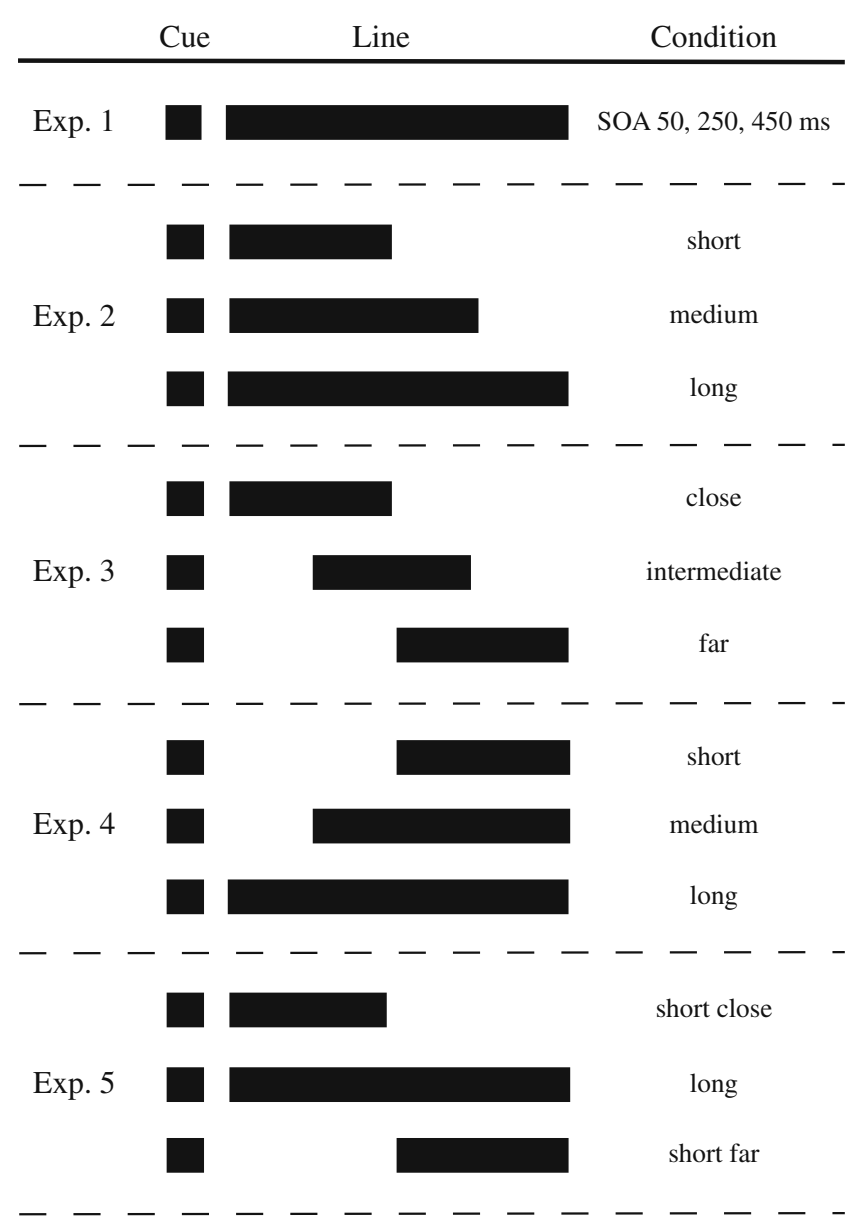

Fig. 3 A comparison of the stimulus types in Experiments 1, 2, 3, 4, and 5. In Experiment 1, the line is always the same length, and distance from the cue is always the same. In Experiment 2, the line varies in length but the near end of the line is always the same distance from the cue. In Experiment 3, the line is always the same length, but the distance of the line from the cue varies. In Experiment 4 , the line varies in length but the far end of the line is always the same distance from the cue. In Experiment 5, the line varies in length and in the distance of the near and far ends from the cue

significant. Length was highly significant, $F(2,36)=7.42$, $M S E=1.16, p<.003$, and least-squares comparisons revealed that long lines $(M=5.08)$ and medium lines $(M=$ 4.64) were each rated as faster than short lines $(M=4.13)$, and that long lines were rated as marginally faster than medium lines.

Direction Ratings of perceived direction were analyzed in a 2 (cue) $\times 3$ (length) repeated measures ANOVA. Cue was significant, $F(1,18)=12.08, M S E=23.34, p<.003$; when cues were on the left, lines were more likely to be rated as expanding from the left $(M=2.49)$, and when cues were on the right, lines were more likely to be rated as expanding from the right $(M=5.64)$. Neither length, $F(2,36)=1.19$, $M S E=0.17, p>.31$, nor Cue $\times$ Length, $F(2,36)=1.06$, $M S E=0.49, p>.35$, was significant. 

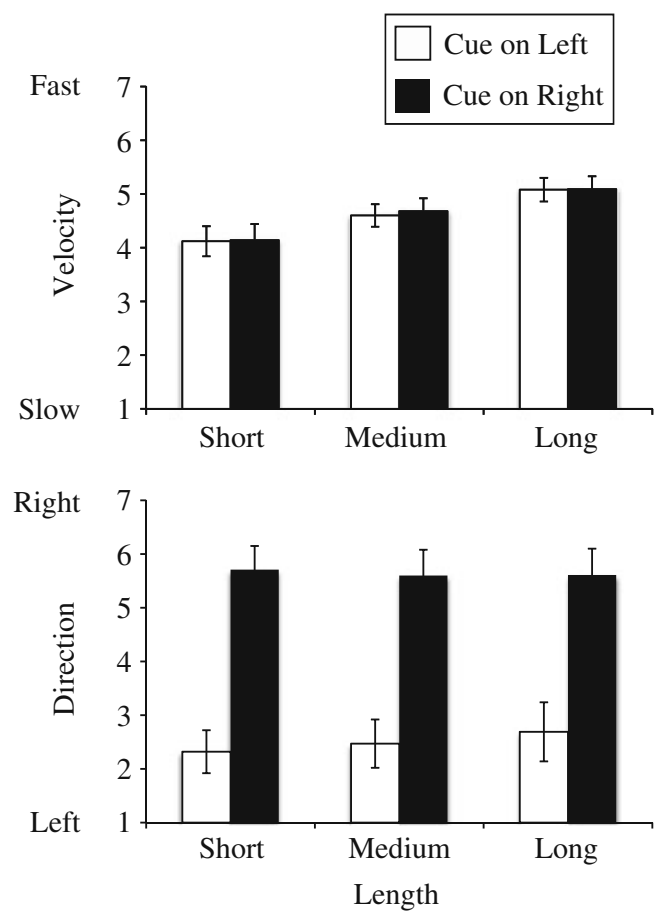

Fig. 4 The ratings of velocity and direction as a function of line length in Experiment 2. Data for velocity ratings are shown in the top panel, and data for direction ratings are shown in the bottom panel. Data when the cue is on the left or right are indicated by open and filled columns, respectively. Error bars reflect standard errors of the means

Ratings of perceived direction for each condition were tested against a null rating of 4 (Bonferroni correction of $.05 / 6=.0083)$. When cues were on the left for short lines $(M=2.32), t(18)=-4.21, p<.0005$, medium lines $(M=$ 2.47), $t(18)=-3.42, p<.003$, and long lines $(M=2.69)$, $t(18)=-2.41, p<.03$, ratings were significantly or marginally less than 4 , indicating that the lines were perceived as expanding from the left. When cues were on the right for short lines $(M=5.71), t(18)=3.93, p<.001$, medium lines $(M=5.59), t(18)=3.33, p<.004$, and long lines $(M=5.61), t(18)=3.28, p<.005$, ratings were significantly greater than 4 , indicating that lines were perceived as expanding from the right.

\section{Discussion}

Ratings of perceived velocity increased with increases in the length of the line. This is consistent with (1) the possibility that ILM is of limited duration, as the perceived velocity of ILM resulting from a given SOA would have to be relatively faster to accomplish the unfolding of a long line than to accomplish the unfolding of a short line, and (2) an attentional-gradient account. Ratings of perceived direction and strength were not influenced by the length of the line. However, Christie and Klein's (2005) notion regarding drawing speed and the strength of ILM predicts that the line length with the slowest velocity of ILM should exhibit the highest strength of ILM; given that the short line received the lowest ratings of perceived velocity, the short line should have also received the most extreme ratings of perceived direction. Alternatively, an attentional-gradient account would predict that a long line would have a larger gradient than would a short line, so the long line should have received more extreme ratings of perceived direction than the short line. Neither of these predicted patterns occurred, as the Cue $\times$ Length interaction in ratings of perceived direction did not approach significance. When line length is varied, there appears to be a dissociation between perceived strength of expansion in a specific direction and perceived relative velocity of that expansion, and this dissociation is not consistent with an attentional-gradient account.

\section{Experiment 3}

The results of Experiment 2 suggest that the perceived velocity of ILM was relatively faster for longer lines. However, in Experiment 2 there was a confound between length of a line and distance of the far end of that line from the cue. It could be argued that increases in ratings of perceived velocity of ILM with increases in line length in Experiment 2 were not due to the increases in line length per se, but rather to increases in the distance of the far end of the line from the cue. If so, increasing the distance between the cue and the far end of a line of constant length (thus increasing distance between the cue and the near end of the line) might have the same effect on ratings of perceived velocity as keeping the near end of the line a constant distance from the cue and increasing the length of the line; that is, for a line of constant length, increasing the distance between the cue and the far end of the line should increase ratings of the perceived velocity of ILM. Accordingly, Experiment 3 presented a line of a constant length that was located at a relatively close, intermediate, or far distance from the cue. If the distance of the far end of the line was the critical parameter in Experiment 2, lines at a far distance should lead to higher ratings of perceived velocity than would lines at a close distance. Alternatively, if the length of the line was the critical parameter in Experiment 2, there should be no differences between the ratings of perceived velocity of the lines far from the cue and the ratings of perceived velocity of the lines close to the cue.

Method

Participants The participants were 21 undergraduates from the same participant pool used in Experiment 1, and none had participated in Experiment 1 or 2. 
Apparatus The apparatus was the same as in Experiment 1.

Stimuli The cue and line stimuli were the same as in Experiment 1, with the following exceptions: On all trials, the SOA was $250 \mathrm{~ms}$. The length of the line on all trials was 96 pixels $\left(3.98^{\circ}\right.$; same as for the short line in Experiment 2). The distance between the closest vertical edge of the cue and the closest vertical edge of the line was close (20 pixels, $0.83^{\circ}$; same as in Experiment 1$)$, intermediate (70 pixels, $\left.2.91^{\circ}\right)$, or far $\left(120\right.$ pixels, $\left.4.98^{\circ}\right)$. Also, the distance of the far end of the line from the cue for the close, intermediate, and far distances was the same as the distance of the far end of the short, medium, and long lines, respectively, from the cue in Experiment 2. The length of the line was the same across trials, but the distance of the near end of the line and the distance of the far end of the line from the cue varied across trials (see Fig. 3). The rating scales were the same as in Experiment 1. In each block, each participant received 42 trials $(2$ [cue: left, right] $\times 3$ [distance: close, intermediate, far] $\times 7$ [replications]) in a different random order.

Procedure The procedure was the same as in Experiment 2.

\section{Results}

Ratings of perceived velocity are shown in the top panel of Fig. 5, and ratings of perceived direction are shown in the bottom panel of the figure. Preliminary analyses revealed that block order did not influence ratings, so subsequent analyses were collapsed across block orders.

Velocity Ratings of perceived velocity were analyzed in a 2 (cue) $\times 3$ (distance) repeated measures ANOVA. Neither cue, $F(1,20)=0.10, M S E=0.39, p>.75$, Cue $\times$ Distance, $F(2,40)=0.17, M S E=0.37, p>.79$, nor distance, $F(2,40)=0.31, M S E=1.15, p>.73$, was significant.

Direction Ratings of perceived direction were analyzed in a 2 (cue) $\times 3$ (distance) repeated measures ANOVA. Cue was significant, $F(1,20)=22.54, M S E=21.08, p<.0001$; when cues were on the left, lines were more likely to be rated as expanding from the left $(M=2.23)$, and when cues were on the right, lines were more likely to be rated as expanding from the right $(M=6.11)$. Neither Cue $\times$ Distance, $F(2,40)=$ $1.86, M S E=1.02, p>.16$, nor distance, $F(2,40)=0.97$, $M S E=0.63, p>.38$, was significant.

Ratings of perceived direction for each condition were tested against a null rating of 4 (Bonferroni correction of $.05 / 6=.0083)$. When cues were on the left for the 20-pixel $(M=2.13), t(21)=-4.42, p<.0003,70-$ pixel $(M=2.32)$, $t(21)=-3.72, p<.0014$, and 120-pixel distances $(M=$
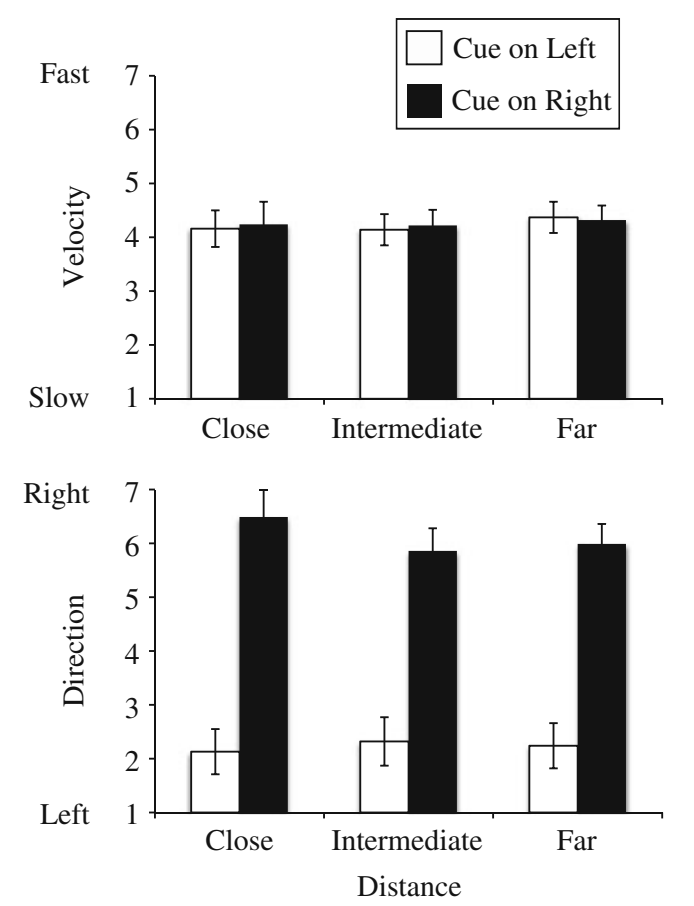

Fig. 5 The ratings of velocity and direction as a function of cue distance in Experiment 3. Data for velocity ratings are shown in the top panel, and data for direction ratings are shown in the bottom panel. Data when the cue is on the left or right are indicated by open and filled columns, respectively. Error bars reflect standard errors of the means

2.24), $t(21)=-4.25, p<.0004$, ratings were significantly less than 4 , indicating that lines were perceived as expanding from the left. When cues were on the right for the 20-pixel $(M=6.49), t(21)=4.03, p<.007,70$-pixel $(M=5.86), t(21)=4.47, p<.0002$, and 120-pixel distances $(M=5.59), t(21)=5.38, p<.0001$, ratings were significantly greater than 4 , indicating that lines were perceived as expanding from the right.

\section{Discussion}

Neither ratings of perceived velocity nor ratings of perceived direction were influenced by the distance of the line from the cue in Experiment 3. The lack of a distance effect on ratings of perceived velocity in Experiment 3 suggests that the length of the line rather than the distance of the far end of the line from the cue was the key parameter in the effect of length on ratings of perceived velocity in Experiment 2. Lack of an effect of distance on ratings of perceived velocity when SOA was constant in Experiment 3, coupled with a significant effect of SOA on ratings of perceived velocity when distance was constant in Experiment 1, does not support the speculation from Experiment 1 that perceived velocity of ILM reflects the velocity required to complete movement from the cue to the 
line during a duration equal to the SOA. The lack of a distance effect suggests that ILM did not originate with the cue or that ILM was insensitive to the distance between the cue and the line; these possibilities do not appear consistent with an attentional-gradient account, although the latter possibility is consistent with Hikosaka, Miyauchi, and Shimojo's (1993b) suggestion that attention in ILM is directed to an object and not to empty space. Similarly, given that the strength of apparent motion is influenced by distance, the lack in Experiment 3 of an effect of distance on ratings of perceived velocity or of an interaction between cue and distance on ratings of perceived direction is not consistent with an apparent-motion account. Also, the lack of a distance effect is consistent with Hamm and Klein's (2002) finding of equal facilitation in judgments of targets at the near end and the far end of a line.

\section{Experiment 4}

The results of Experiment 3 suggest that the effects of length in Experiment 2 did not result from differences in the distance of the far end of the line from the cue, but rather resulted from differences in the length of the line. Such a conclusion leads to a prediction that if the distance of the far end of a long line from the cue is equal to the distance of the far end of a short line from the cue, then the long line would be rated as expanding at a relatively faster perceived velocity than would the short line. Accordingly, in Experiment 4, the distance of the far end of the line from the cue was constant, and the length of the line and the distance of the near end of the line from the cue were varied. If ILM is influenced by the length of the line, the perceived velocity of a long line close to the cue should be rated as faster than the perceived velocity of a short line far from the cue. Also, given that the distance of the near end of the line from the cue differed across close, intermediate, and far lines in Experiment 3, but that no differences in ratings of perceived velocity were found in that experiment, any differences in the ratings of velocity of ILM in Experiment 4 should be due to the length of the line rather than to differences in the distance of the near end of the line from the cue.

Method

Participants The participants were 16 undergraduates from the same participant pool used in Experiment 1, and none had participated in the previous experiments.

Apparatus The apparatus was the same as in Experiment 1.

Stimuli The cue and line stimuli were the same as in Experiment 2, with the following exceptions: The distance between the closest edge of the cue and the closest edge of the line for the short line was 120 pixels $\left(4.98^{\circ}\right.$; same as for the far line in Experiment 3), for the medium line was 70 pixels $\left(2.91^{\circ}\right.$; same as for the intermediate line in Experiment 3$)$, and for the long line was 20 pixels $\left(0.83^{\circ}\right.$; same as for the close line in Experiment 3). As a result, the distance of the far end of the line from the cue was 216 pixels $\left(8.96^{\circ}\right.$; same as for the long line in Experiment 2) for all lines, but the length of the line and the distance between the near end of the line and the cue varied across lines (see Fig. 3). Rating scales were the same as in Experiment 1. In each block, each participant received 42 trials (2 [cue: left, right] $\times 3$ [length: short, medium, long] $\times 7$ [replications]) in a different random order.

Procedure The procedure was the same as in Experiment 2.

Results

Ratings of perceived velocity are shown in the top panel of Fig. 6, and ratings of perceived direction are shown in the bottom panel of the figure. Preliminary analyses revealed that block order did not influence ratings, so subsequent analyses were collapsed across block orders.
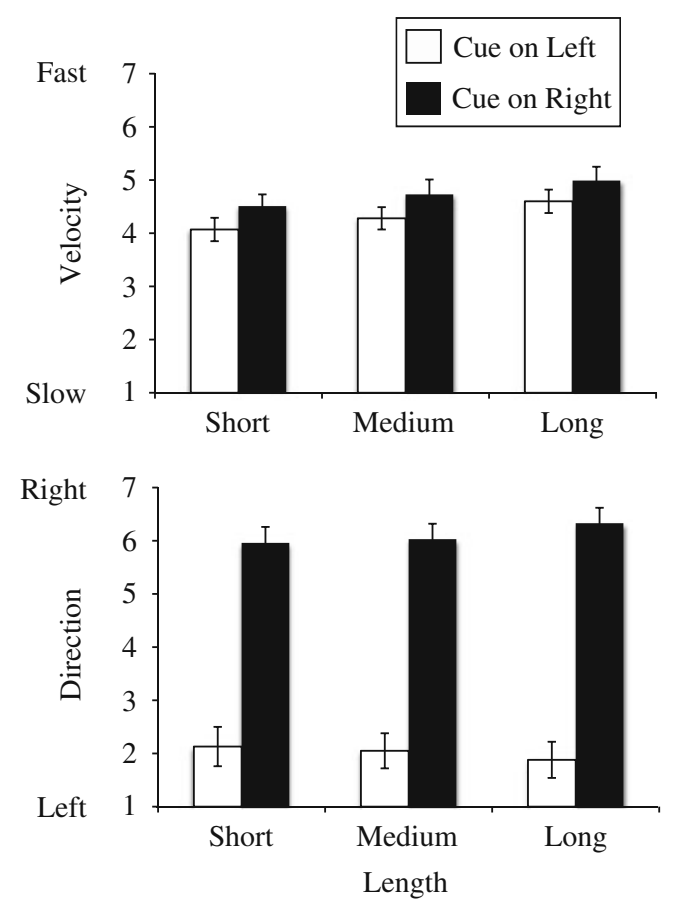

Fig. 6 The ratings of velocity and direction as a function of line length in Experiment 4. Data for velocity ratings are shown in the top panel, and data for direction ratings are shown in the bottom panel. Data when the cue is on the left or right are indicated by open and filled columns, respectively. Error bars reflect standard errors of the means 
Velocity Ratings of perceived velocity were analyzed in a 2 (cue) $\times 3$ (length) repeated measures ANOVA. Length was significant, $F(2,30)=4.24, M S E=0.48, p<.003$, and least-squares comparison revealed that long lines $(M=$ 4.80) were rated as faster than short lines $(M=4.29)$ and that medium lines $(M=4.51)$ did not differ from long or from short lines. Cue was significant, $F(1,15)=6.48$, $M S E=0.68, p<.03$; when cues were on the left, velocity was rated as slower $(M=4.32)$ than when cues were on the right $(M=4.74)$. Cue $\times$ Length was not significant, $F(2,30)=0.53, M S E=0.15, p>.94$.

Direction Ratings of perceived direction were analyzed in a 2 (cue) $\times 3$ (length) repeated measures ANOVA. Cue, $F(1,15)=$ 45.75, $M S E=8.77, p<.0001$, and Cue $\times$ Length, $F(2,30)=$ $4.09, M S E=0.23, p<.05$, were significant. When cues were on the left, lines were more likely to be rated as expanding from the left $(M=2.02)$, and when cues were on the right, lines were more likely to be rated as expanding from the right $(M=6.10)$. To explore the Cue $\times$ Length interaction further, a difference score (mean rating for right cue minus mean rating for left cue) was calculated for each length for each participant, and these difference scores were analyzed in a one-way repeated measures ANOVA. Difference score was significant, $F(2,30)=4.08, M S E=1.25, p<.003$, and leastsquares comparison revealed that the 20-pixel $(M=3.81)$ and 70-pixel $(M=3.97)$ lines resulted in smaller difference scores (i.e., less extreme ratings) than did the 120 -pixel $(M=4.47)$ line, and difference scores for the 20- and 70-pixel lines did not differ significantly. As shown in the bottom panel of Fig. 6, the difference in ratings of direction for cues on the left and for cues on the right was slightly larger (i.e., more extreme ratings) for long lines than for short lines. Length was not significant, $F(2,30)=0.20, M S E=0.14, p>.81$.

Ratings of perceived direction for each condition were tested against a null rating of 4 (Bonferroni correction of $.05 / 6=.0083)$. When cues were on the left for short lines $(M=2.13), t(15)=-5.10, p<.0001$, medium lines $(M=2.05), t(15)=-5.98, p<.0001$, and long lines $(M=$ $1.86), t(15)=-6.26, p<.0001$, ratings were significantly less than 4 , indicating that lines were perceived as expanding from the left. When cues were on the right for short lines $(M=5.96), t(15)=6.48, p<.0001$, medium lines $(M=6.03), t(15)=7.06, p<.0001$, and long lines $(M=6.33), t(15)=8.14, p<.005$, ratings were significantly greater than 4 , indicating that lines were perceived as expanding from the right.

\section{Discussion}

Ratings of perceived velocity increased with increases in line length in Experiment 4, and this is consistent with increases in ratings of perceived velocity with increases in line length in Experiment 2. A comparison of the ratings of perceived velocity in Experiments 3 and 4 suggests that differences in perceived velocity are not systematically related to the distance of the near end of the line, and a comparison of the ratings of perceived velocity in Experiments 2 and 4 suggests that differences in perceived velocity are not systematically related to the distance of the far end of the line. Also, ratings of perceived velocity were relatively slower when the line was perceived to expand from the left than when the line was perceived to expand from the right. A direction effect on ratings of perceived velocity was not found in Experiments 1, 2, and 3; the reason for this finding in Experiment 4 is not clear, and this difference is probably not reliable. Ratings of perceived direction were more extreme for long lines than for short lines; however, long lines were rated as relatively faster than short lines, and this initially appears inconsistent with suggestions in Christie and Klein (2005) and in Experiment 1 that stronger ILM is linked with a slower perceived velocity of ILM. The effect in Experiment 4 was significant only at the .05 level, and the analogous effect in Experiment 2 did not approach significance. It might be that strength of ILM was more salient or apparent in Experiment 4 than in Experiment 2, because in Experiment 4 the ending point of expansion (the far end of the line) was at the same location for different line lengths.

\section{Experiment 5}

The results of Experiments 2, 3, and 4 suggested that ratings of perceived velocity in ILM were related to the length of the line and not to the distance of the near or the far end of the line from the cue. Such a conclusion leads to predictions that (1) a long line should result in ratings of faster perceived velocity than should a short line when the near ends of the long line and the short line are the same distance from the cue (replicating Experiment 2); (2) ratings of perceived velocity of lines of a constant length but located at different distances from the cue should not differ (replicating Experiment 3); and (3) a long line should result in ratings of faster perceived velocity than should a short line when the far ends of the long line and the short line are the same distance from the cue (replicating Experiment 4). Accordingly, in Experiment 5 a long line, a short close line, and a short far line were presented. The near end of the short close line was the same distance from the cue as was the near end of the long line, and the far end of the short far line was the same distance from the cue as was the far end of the long line. By using such a design, Experiment 5 attempted to replicate with a within-subjects design the 
salient findings from the between-subjects comparisons across Experiments 2, 3, and 4.

\section{Method}

Participants The participants were 17 undergraduates from the same participant pool used in Experiment 1, and none had participated in the previous experiments.

Apparatus The apparatus was the same as in Experiment 1.

Stimuli The cue and line stimuli were the same as in Experiment 1, with the following exceptions: On all trials, the SOA was $250 \mathrm{~ms}$. The lengths of the short close line and the short far line were 96 pixels $\left(3.98^{\circ}\right.$; same as for the short lines in Experiments 2 and 4 and all lines in Experiment 3), and the length of the long line was 196 pixels $\left(8.13^{\circ}\right.$; same as for the long lines in Experiments 2 and 4). The distances of the near end of the short close line and the near end of the long line from the cue were 20 pixels $\left(0.83^{\circ}\right.$; same as for the near ends of all lines in Experiment 2, the close line in Experiment 3, and the long line in Experiment 4), and the distance of the near end of the short far line from the cue was 120 pixels $\left(4.98^{\circ}\right.$; same as for the far line in Experiment 3 and the short line in Experiment 4). The distance of the far end of the short close line from the cue was 116 pixels $\left(4.81^{\circ}\right.$; same as for the far ends of the short line in Experiment 2 and the close line in Experiment 3), and the distances of the far ends of the long line and the short far line from the cue were 216 pixels $\left(8.96^{\circ}\right.$; same as for the far ends of the long line in Experiment 2, the far line in Experiment 3, and all lines in Experiment 4) (see Fig. 3). Rating scales were the same as in Experiment 1. In each block, each participant received 42 trials $(2$ [cue: left, right] $\times 3$ [length: short close, short far, long] $\times 7$ [replications]) in a different random order.

Procedure The procedure was the same as in Experiment 2.

\section{Results}

Ratings of perceived velocity are shown in the top panel of Fig. 7, and ratings of perceived direction are shown in the bottom panel of the figure. Preliminary analyses revealed that block order did not influence ratings, so subsequent analyses were collapsed across block orders.

Velocity Ratings of perceived velocity were analyzed in a 2 (cue) $\times 3$ (length) repeated measures ANOVA. Length was significant, $F(2,32)=9.11, M S E=0.75, p<.008$, and least-squares comparisons revealed that long lines $(M=$ 4.91) were rated as faster than short close lines $(M=4.14)$

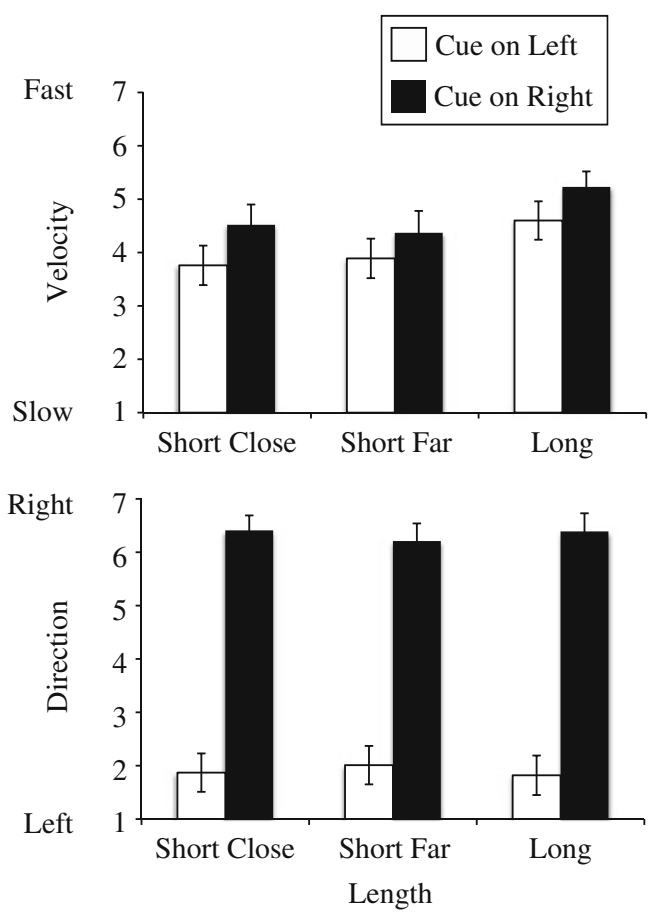

Fig. 7 The ratings of velocity and direction as a function of line length in Experiment 5. Data for velocity ratings are shown in the top panel, and data for direction ratings are shown in the bottom panel. Data when the cue is on the left or right are indicated by open and filled columns, respectively. Error bars reflect standard errors of the means

or short far lines $(M=4.13)$, and that short close lines and short far lines were not significantly different. Neither cue, $F(1,16)=1.91, M S E=5.16, p>.18$, nor Cue $\times$ Length, $F(2,32)=0.36, M S E=0.47, p>.70$, was significant.

Direction Ratings of perceived direction were analyzed in a 2 (cue) $\times 3$ (length) repeated measures ANOVA. Cue was significant, $F(1,16)=48.63, M S E=10.32, p<.0001$; when cues were on the left, lines were more likely to be rated as expanding from the left $(M=1.90)$, and when cues were on the right, lines were more likely to be rated as expanding from the right $(M=6.34)$. Neither Cue $\times$ Length, $F(2,32)=2.12$, $M S E=0.17, p>.13$, nor length, $F(2,40)=0.18, M S E=0.07$, $p>.83$, was significant.

Ratings of perceived direction for each condition were tested against a null rating of 4 (Bonferroni correction of $.05 / 6=.0083)$. When cues were on the left for short close lines $(M=1.87), t(16)=-5.92, p<.0001$, short far lines $(M=2.01), t(16)=-5.49, p<.0001$, and long lines $(M=1.82), t(16)=-5.85, p<.0001$, ratings were significantly less than 4 , indicating that lines were perceived as expanding from the left. When cues were on the right for short close lines $(M=6.41), t(16)=8.55$, $p<.0001$, short far lines $(M=6.21), t(16)=6.64, p<.004$, and long lines $(M=6.39), t(16)=7.04, p<.0001$, ratings 
were significantly greater than 4 , indicating that lines were perceived as expanding from the right.

\section{Discussion}

Ratings of perceived velocity were higher for the long line than for either the short close line or the short far line, and ratings of perceived velocity did not differ between the short close line and the short far line. The former difference replicates the effect of length in Experiments 2 and 4 and is consistent with the hypothesis that perceived velocity of ILM is influenced by line length, and the latter lack of difference replicates the lack of an effect of distance in Experiment 3 and is consistent with the hypothesis that the distance of the near or the far end of the line from the cue does not influence the perceived velocity of ILM. Unlike in Experiment 4, in Experiment 5 the Cue $\times$ Length interaction was not significant in ratings of perceived direction; more specifically, in Experiment 5 length did not influence the perceived strength of motion (in a specific direction) in ILM. It should be noted that two of the three levels in the length variable in Experiment 5 included stimuli of a constant length that differed in distance (short close, short far), so the lack of an effect of length in Experiment 5 is consistent with the lack of an effect of distance in Experiment 3, as well. The inclusion of differences in distance and the confounding of distance and length across lines in Experiment 5 might have introduced additional noise that diluted any possible Cue $\times$ Length interaction. Even so, the lack of effects of length and distance on the strength of ratings of perceived direction of ILM in Experiment 5 is generally consistent with the findings in Experiments 2, 3, and 4.

\section{General discussion}

The primary empirical findings were that ratings of (1) perceived velocity of ILM decreased with increases in the SOA between appearance of the cue and appearance of the line from 50 to $250 \mathrm{~ms}$ and from 250 to $450 \mathrm{~ms}$; (2) perceived strength of ILM (i.e., extremity of ratings of perceived direction) increased with increases in SOA from 50 to either 250 or $450 \mathrm{~ms}$; (3) perceived velocity of ILM increased with increases in the length of the line; (4) perceived strength of ILM were not influenced by increases in the length of the line; (5) perceived velocity of ILM and perceived strength of ILM were not influenced by increases in the distance of the near or the far end of the line from the cue; and (6) perceived velocity of ILM and perceived strength of ILM were generally not influenced by whether
ILM was leftward or rightward. In summary, and within the range of parameters used here, (a) increases in SOA decreased ratings of perceived velocity and increased the extremity (strength) in ratings of perceived direction; (b) increases in line length increased ratings of perceived velocity and did not influence the strength of ratings of perceived direction; and (c) increases in distance of the line from the cue did not influence ratings of perceived velocity or the strength in ratings of perceived direction. Perceived velocity of ILM is not solely a function of the strength of illusory motion, and perceived strength of ILM is not solely a function of the velocity of illusory motion.

There is considerable consistency in the ratings of perceived velocity of ILM for similar stimuli across experiments. Ratings for the short far line and for the long line in Experiment 5 were similar to ratings for the short line and for the long line, respectively, in Experiment 4, and in both Experiments 4 and 5 the long line was rated as expanding at a relatively faster velocity. Similarly, ratings for the short close line and for the long line in Experiment 5 were similar to ratings for the short line and for the long line, respectively, in Experiment 2, and in both Experiments 2 and 5 the long line was rated as expanding at a relatively faster velocity. Ratings for the long line in Experiment 5 were similar to ratings for the long line in Experiments 2 and 4, and in Experiments 2, 4, and 5 the long line was rated as expanding at a relatively faster velocity. Also, ratings for the short close line and for the short far line in Experiment 5 were similar to the ratings for the close line and for the far line, respectively, in Experiment 3, and in neither Experiment 3 nor 5 were there differences in ratings of perceived velocity between these lines. There is also consistency in ratings of perceived direction of ILM for similar stimuli across experiments. As would be expected, in Experiments 1, 2, 3, 4, and 5, motion was perceived as expanding from the left when the cue was on the left and as expanding from the right when the cue was on the right. Length of the line and distance of the line from the cue generally did not influence the extremity (strength) of perceived direction ratings.

The data are relevant for a consideration of the relationship of attention and ILM. The results of Experiments 1, 2, 3, 4, and 5 do not appear completely consistent with the notion that ILM results from differences in a static distribution of attention in which different portions of the line receive different and unchanging allocations of attention (i.e., in which there is an attentional gradient along the length of the line). Even so, the lack of an effect of distance of the line from the cue is consistent with Hikosaka et al.'s (1993b) suggestion that attention is allocated to objects and not to empty space. Given that longer lines were consistently rated as exhibiting a faster 
relative perceived velocity than were slower lines, it could be suggested that the faster relative perceived velocity of longer lines reflects a larger attentional gradient between the cue and the far end of the line for longer lines. However, the presence of a larger attentional gradient should also lead to ratings of greater relative strength for longer lines, and although this effect was observed in Experiment 4, it was not observed in Experiments 2 and 5. Perhaps more critically, if a static attentional gradient is the mechanism of ILM, then SOA between the appearance of the cue and the appearance of the line should not influence the perceived velocity or strength of ILM. However, in Experiment 1 increases in SOA decreased ratings of perceived velocity and increased the strength (extremity of ratings of perceived direction) of ILM.

The results of Experiments 1, 2, 3, 4, and 5 appear more consistent with a notion that attention is dynamically shifted than with a notion that attention is statically distributed. If attention is dynamic and either leads or follows ILM, differences in the relative perceived velocity or perceived strength of ILM as a function of SOA or as a function of line length might then be accounted for (e.g., a long SOA would allow more of the shift to an anticipated line to be completed before the line actually appeared; a smaller remaining shift after the line appeared would cover less distance and so might be perceived as a slower velocity). In Experiments 2, 4, and 5, the effects of length on ratings of perceived velocity suggest that attention is expanded along the line following ILM, as shifting a longer distance with a constant and brief duration of ILM would necessitate a faster velocity. Furthermore, the lack of an effect of distance in Experiment 3 and the similarity of ratings for lines of different length but similar far-end distances in Experiment 4 suggests that such shifts are not discrete shifts in which attention is disengaged from the cue and then reengaged at the far end of the line, but rather are analogue shifts along the length of the line (consistent with expansion of a zoom lens of attention; cf. Hamm \& Klein, 2002). Comparisons of Experiments 2, 3, 4, and 5 suggest that such shifts traverse the length of the line but do not traverse the empty space between the cue and the line; however, it is not clear why such an analogue shift of attention would be influenced by whether that shift crossed empty space or crossed a line.

The data are relevant for a consideration of the relationship of apparent motion and ILM. Studies of normative apparent motion suggest that the strength of apparent motion is influenced by the spatial distance between the stimuli and by the ISI or SOA between the appearance of each of the stimuli, and in general, longer ISIs or SOAs are required as spatial separation increases (e.g., Farrell, 1983). Thus, a change in either distance (length) or SOA without a compensating change in the other parameter should have influenced the strength of any apparent motion in Experiments 1, 2, 3, 4, and 5, and if ILM is based on apparent motion, such a change should have influenced the strength of ILM as well. In Experiment 1, distance between the cue and the line was constant but SOA varied, and the increase in strength of ratings of perceived direction (i.e., increase in the extremity of ratings) with the 250- and $450-\mathrm{ms} \mathrm{SOAs}$ was consistent with an apparent motion account. However, SOA was constant in Experiments 2, 3, 4, and 5, but differences in the distance of the near end of the line from the cue or in the distance of the far end of the line from the cue did not systematically influence the strength of ratings of perceived direction. Along these lines, if apparent motion bridges the gap between the cue and the line, the effect of SOA in Experiment 1 predicts that lines closer to the cue in Experiments 3, 4, and 5 should have been rated as expanding at a relatively faster velocity than were lines farther from the cue, but such an effect was not found.

Accounts of a possible relationship between apparent motion and ILM have usually focused on translational apparent motion (involving a change of location). Even though the data do not appear consistent with translational apparent motion, they might be more consistent with a transformational apparent motion (involving a change of shape). A mechanism based on transformational apparent motion suggests that ILM results from changes in shape (e.g., a square cue extending on one side to become an elongated rectangle) rather than from changes in location. Indeed, Figure 9 in Tse and Logothetis's (2002) discussion of transformational apparent motion shows two stimuli similar to those in the present Experiment 4, in which the far end of the line is a consistent distance from the cue but the length of the line varies. Tse and Logothetis speculated that the perceived velocity of expansion should be faster for a square transforming into a relatively longer line than into a relatively shorter line, and such a speculation is in line with the findings in the present Experiments 2, 4, and 5. However, in Tse and Logothetis's stimuli, as well as in some (e.g., Downing \& Treisman, 1997) but not other (e.g., Hikosaka et al., 1993a) examples of ILM, the line did not spatially overlap the position of the cue, and there was a clear spatial gap between the cue and the line. How the presence of a spatial gap between the cue and the line might influence processes in transformational apparent motion that might contribute to ILM is not clear.

The data provide critical information regarding (1) perceived velocity of ILM, (2) perceived strength of ILM, and (3) the relationship between the perceived velocity and perceived strength of ILM. Ratings of perceived velocity were influenced by SOA and by line length, but ratings of perceived velocity were not influenced by distance of the line from the cue. Ratings of perceived direction and strength were influenced by whether the cue was on the left 
or right and by an interaction of SOA with whether the cue was on the left or right, but ratings of perceived direction and strength were generally not influenced by line length or by distance of the line from the cue. The patterns of ratings of perceived velocity and ratings of perceived direction and strength across the experiments were not fully consistent with an attentional-gradient or an apparent-motion account of ILM. It might be objected that the rating scales were insensitive to differences that would have supported an attentional-gradient or an apparent-motion account of ILM; however, strong effects of cue in ratings of perceived direction, systematic effects of length on ratings of perceived velocity, the use of seven response categories in each scale, and the general consistency of ratings across the experiments do not offer strong support for such a possibility. Overall, differences in ratings across the experiments suggest that a faster perceived velocity of ILM is perceived with slower drawing speeds or longer lines and that stronger ILM is perceived with SOAs of a few hundred milliseconds (i.e., with slower perceived illusory velocities); these findings offer useful constraints for any future theory of ILM.

Author Note The authors thank William Davis, Jennifer Grabowski, Dalvina Green, and Kristen McCall for assistance in data collection.

\section{References}

Bavelier, D., Schneider, K. A., \& Monacelli, A. (2002). Reflexive gaze orienting induces the line-motion illusion. Vision Research, 42, 2817-2827.

Christie, J., \& Klein, R. M. (2005). Does attention cause illusory line motion? Perception \& Psychophysics, 67, 1032-1043.

Downing, P. E., \& Treisman, A. M. (1997). The line-motion illusion: Attention or impletion? Journal of Experimental Psychology: Human Perception and Performance, 23, 768-779.

Eagleman, D. M., \& Sejnowski, T. J. (2003). The line-motion illusion can be reversed by motion signals after the line disappears. Perception, 32, 963-968.

Eriksen, C. W., Pan, K., \& Botella, J. (1993). Attentional distribution in visual space. Psychological Research, 56, 5-13.

Farrell, J. E. (1983). Visual transformations underlying apparent movement. Perception \& Psychophysics, 33, 85-92.

Faubert, J., \& von Grünau, M. (1995). The influence of two spatially distinct primers and attribute priming on motion induction. Vision Research, 35, 3119-3130.
Fuller, S., \& Carrasco, M. (2009). Perceptual consequences of visual performance fields: The case of the line motion illusion. Journal of Vision, 9(4), 13:1-17.

Hamm, J. P., \& Klein, R. M. (2002). Does attention follow the motion in the "shooting line" illusion? Perception \& Psychophysics, 64, 279-291.

Hikosaka, O., Miyauchi, S., \& Shimojo, S. (1993a). Focal visual attention produces illusory temporal order and motion sensation. Vision Research, 33, 1219-1240.

Hikosaka, O., Miyauchi, S., \& Shimojo, S. (1993b). Visual attention revealed by an illusion of motion. Neuroscience Research, 1, $11-18$.

Hofmans, J., Theuns, P., \& Mairesse, O. (2007). Impact of the number of response categories on linearity and sensitivity of "selfanchoring scales": A functional measurement approach. Methodology, 3, 160-169.

Kanizsa, G. (1951). Sulla polarizzazione del movimento gamma. Archivio di Psicologia, Neurologia e Psichiatria, 3, 224-267.

Kawahara, J., Yokosawa, K., Nishida, S., \& Sato, T. (1996). Illusory line motion in visual search: Attentional facilitation or apparent motion. Perception, 25, 901-920.

Scharlau, I., \& Horstmann, G. (2006). Perceptual latency priming and illusory line motion: Facilitation by gradients of attention? Advances in Cognitive Psychology, 2, 87-97.

Schmidt, W. (2000). Endogenous attention and illusory line motion reexamined. Journal of Experimental Psychology: Human Perception and Performance, 26, 980-996.

Shimojo, S., Hikosaka, O., \& Miyauchi, S. (1999). Automatic and controlled attention detected by the line motion effect. In D. Gopher \& A. Koriat (Eds.), Attention and performance XVII: Cognitive regulation of performance. Interaction of theory and application (pp. 145-163). Cambridge: MIT Press.

Shimojo, S., Miyauchi, S., \& Hikosaka, O. (1997). Visual motion sensation yielded by non-visually driven attention. Vision Research, 37, 1575-1580.

Spence, C., \& Parise, C. (2010). Prior entry: A review. Consciousness and Cognition, 19, 364-379.

Tse, P. U., \& Logothetis, N. K. (2002). The duration of 3-D form analysis in transformational apparent motion. Perception \& Psychophysics, 64, 244-265.

von Grünau, M., Dubé, S., \& Kwas, M. (1996). Two contributions to motion induction: A preattentive effect and facilitation due to attentional capture. Vision Research, 36, 2447-2457.

von Grünau, M., \& Faubert, J. (1994). Intraattribute and interattribute motion induction. Perception, 23, 913-928.

von Grünau, M., Racette, L., \& Kwas, M. (1996). Measuring the attentional speed-up in the motion induction effect. Vision Research, 36, 2433-2446.

Weijters, B., Cabooter, E., \& Schillewaert, N. (2010). The effect of rating scale format on response styles: The number of response categories and response category labels. International Journal of Research in Marketing, 27, 236-247. 